\title{
Chern-Simons Field Theory of Two-Dimensional Electrons in the Lowest Landau Level
}

\author{
Lizeng Zhang \\ Department of Physics and Astronomy \\ The University of Tennessee, Knoxville, TN 37996 \\ and Solid State Division, Oak Ridge National Laboratory \\ Oak Ridge, Tennessee 37831
}

\begin{abstract}
We propose a fermion Chern-Simons field theory describing two-dimensional electrons in the lowest Landau level. This theory is constructed with a complete set of states, and the lowest Landau level constraint is enforced through a $\delta$-functional described by an auxiliary field $\lambda$. Unlike the field theory constructed directly with the states in the lowest Landau level, this theory allows one utilizing the physical picture of "composite fermion" to study the fractional quantum Hall states by mapping them onto certain integer quantum Hall states; but unlike it in the unconstrained theory, such a mapping is sensible only when interactions between electrons are present. An "effective mass", which characterizes the scale of low energy excitations in the fractional quantum Hall systems, emerges naturally from our theory. We study a Gaussian effective theory and interpret physically the dressed stationary point equation for $\lambda$ as an equation for the "mass renormalization" of composite fermions.
\end{abstract}

PACS numbers: $73.40 . \mathrm{Hm}$ 


\section{INTRODUCTION}

The fermion Chern-Simons field theoretical approach [1,2], motivated by the composite fermions theory [3] in which the fractional quantum Hall effect (FQHE) [4] is viewed as the integer QHE of certain bound objects of electrons and vortices, i.e, the "composite fermions", has shown to be a useful method for investigating the physical properties of the FQHE. In such a field theoretical approach, one, roughly speaking, "attaches" even number of flux quanta to the two-dimensional (2D) electrons through introducing the so called ChernSimons gauge field. In the mean-field approximation where the statistical gauge fluxes are delocalized from the electrons and uniformly spread out in the 2D plane, this mean gauge field partially cancels the external magnetic field such that at certain filling fractions the mean-field state is descibed by integer number of filled effective Landau level (LL), which signals the occurrence of the FQHE. Qualitatively correct physics of the FQHE may be recovered by incorporating the effect of fluctuations of the gauge field. This approach has been adopted for investigating the FQHE at filling fractions with odd-denominators at zero temperature in [1, [0,6], and at finite temperature in [7]. For the $\nu=1 / 2$ state, in which the mean statistical field and external magnetic field precisely cancel each other such that the mean-field theory is just a filled Fermi sea, this approach [2] has successfully explained the anomaly in the surface acoustic wave propagation [8]. Its qualitative correctness has also been verified by a series of experiments [9].

Since the FQHE is observed in the presence of high magnetic field $B$, it is conceivable that the FQHE may be understood at the infinite magnetic field limit, where the Hilbert space is composed solely by the states in the lowest LL (LLL). This limit is important conceptually, since in which the kinetic energy is frozen, showing clearly the non-perturbative effect of electron-electron interactions. Because the size of the Hilbert space is drastically reduced by the restriction to the LLL, this limit is also of great convenience for numerical studies which have been indispensable to the development of our understanding of the FQHE. The seminal work of Laughlin [10] is formulated entirely within the LLL. Noticeably that Jain's 
wavefunction, whose construction is aided with states in higher LLs (to utilize the mapping between FQH states and IQH states), also needs to be ultimately mapped back to the LLL in order to give the correct low energy physics [3].

The importance of the LLL is not explicit in the present field theoretical approach, at least not in the first order loop expansion commonly adopted in the literature. In fact the electron-electron interaction, which is crucial for the occurrence of the FQHE, plays only a nominal role in the Chern-Simons field theory scheme mentioned above. Formally, if one actually carried out the same calculation for non-interacting electrons at these special fillings (become clear later), one would obtain qualitatively the same physics (e.g., incompressibility of the ground state) as that with interactions. On the positive side, this fact may be interpreted as the "correctness" of the mean-field theory [11]; on the other hand, it is also quite disturbing since we know that the ground state of a system of non-interacting electrons with a partially filled LL is compressible and is highly degenerate, and the FQHE occurs only as a result of strong electron-electron correlations. Because the interaction has not been treated properly, the energy scale of the problem in this approach is incorrectly given by an effective cyclotron energy which is on the same order of the bare cyclotron energy, while the physical energy scale is set by the electron-electron interaction. This serious defect of the theory not only renders any quantitative description of the low energy physics of the FQHE impossible, but has also raised a fundamental question concerning the validity of the theory itself. In [2,5], this problem was dealt phenomenologically through a Fermi liquid theory. In this approach, the magnetic field is treated semi-classically, and the effective mass is introduced as a free parameter. This Fermi liquid approach has been examined recently through a comparison with exact numerical results on small systems [6]. While it provides a way of dealing the problem of mass renormalization, its phenomenological nature is unsatisfactory. Furthermore, for FQH states at filling fraction such as $\nu=1 / 3,1 / 5,2 / 5$ etc. in which the number of filled effective LLs are small, the semi-classical approximation is expected to break down due to the strong effective magnetic field. What we would like to have is a microscopic formalism with a mean-field theory for which minimal fluctuation 
corrections are needed to reveal the qualitatively (and quantitatively) correct physics. With this philosophy in mind, we propose here a fermion Chern-Simons field theory constrained to the LLL, in which the essential role of the electron-electron interaction for the FQHE is explicit. Our hope is that true physics may be revealed in some low order perturbation expansion.

In the LLL, the kinetic energy is frozen and the effect of interaction is non-perturbative. There are several works in the literature dealing with the issue of formulating field theories constrained in the LLL [12, 13]. However, since the states in the LLL are incomplete, a field theory constructed directly from the states in the LLL possesses some peculiar properties which make evaluations of physical properties extremely difficult. Here we shall follow an alternative formalism. To illustrate our approach, it is instructive to have a closer look at Jain's construction of the ground state wavefunction for a FQH state [3]. While the wavefunction itself is built solely from the states in the LLL, it is constructed in such a way that the role of higher LLs is explicit. Mathematically, it provides a compact way of expressing otherwise (i.e., writing directly within the Hilbert space of the LLL) an extremely complicated object. Physically, it helps tremendously in gaining insight (i.e., bridging the connection between the FQHE and the IQHE) to the problem. Our approach here is similar in spirit: instead of working directly in the subspace spanned by the states in the LLL, we shall start with the whole Hilbert space to utilize the notion of mapping the FQHE onto the IQHE. The restriction to the LLL is enforced by a $\delta$-functional described by an auxiliary field $\lambda(r)$ (see below). Thus, just like that in Jain's theory one employs states in the higher LLs to construct the wavefunction but ultimately projects it back to the LLL, here we use the higher LLs in intermediate stages of calculations and integrate out the $\lambda$-field at the end to realize the constraint. As we shall see later, in this theory the density and current fluctuations not only couples to themselves and to each other, but also couples to a local kinetic energy density such that the constraint to the LLL is satisfied.

A direct consequence of this approach is to provide a microscopic calculation of the energy gap, which is experimentally measurable $4,14,15$ and is an important quantity for 
estimating the effect of thermal fluctuations on the accuracy of the FQHE. One may also study the low energy collective excitation spectrum within the present theory. The properties of low energy excitations may be extrapolated from numerical results obtained through exact diagonalization on small systems [4] and from direct construction of trial wavefunctions for low energy excited states [3, [4, 10, [6], or be studied through the Chern-Simons-LandauGinzburg field theory [17]. While these studies are very useful to our understanding of the physics of the FQHE, for quantitative comparisons with experiments we still need a microscopic calculation done directly in the thermodynamic limit, especially in view of the current discrepancies between experiments and theories on the physical quantities such as the magnitude of the energy gap. The previous works using the unconstrained fermion Chern-Simons field theory [1,0] cannot serve this purpose owing to its intrinsic difficulty with the energy scale discussed above.

In the present work, we shall consider only those systems with filling fraction $\nu=n /(1+$ $2 p n$ ) ( $p, n$ integer) whose ground states are known to be incompressible and for whom there are considerable amount of numerical results available which may be helpful for testing the correctness of our theory. On the other hand, computations, especially the numerical solution for the dressed stationary point equation of the $\lambda$-field in such a system are unfortunately much more complicated than that for a $\nu=1 / 2 p$ system in which the mean-field ground state is just a filled fermi sea. However, since the calculation of the $\nu=1 / 2 p$ state is quite different from the present one, it shall be studied in a separate work.

The rest of the paper is organized as following: in the next section we present the formalism of our approach; in section III, we study the electro-magnetic response; we discuss some consequences of the theory and conclude in the section IV.

\section{FORMALISM}

The quantum many-body wave function describing a system of $2 \mathrm{D}$ electrons of band mass $m_{b}$ in a magnetic field $\vec{B}=\vec{\nabla} \times \vec{A}$ occupying only the LLL satisfies the condition 


$$
\frac{1}{2 m_{b}}\left(-i \hbar \vec{\nabla}_{j}+\frac{e}{c} \vec{A}\right)^{2} \psi\left(\vec{r}_{1}, \ldots, \vec{r}_{j}, \ldots, \vec{r}_{N}\right)=\frac{1}{2} \hbar \omega_{c} \psi\left(\vec{r}_{1}, \ldots, \vec{r}_{j}, \ldots, \vec{r}_{N}\right), \quad \forall j
$$

where $\omega_{c}=e B / m_{b} c$ is the cyclotron frequency. In the second quantized language, one may express the partition function for such a system by a coherent functional integral subjected to a constraint:

$$
\mathcal{Z}=\int \mathcal{D} \psi^{\dagger} \mathcal{D} \psi \mathcal{D} \lambda e^{-S}
$$

with the action (in the unit $\hbar=1$ )

$$
\begin{aligned}
S= & \int_{0}^{\beta} d \tau \int d^{2} r\left\{\psi^{\dagger}\left(\partial_{\tau}-\mu\right) \psi(r)+\frac{1}{2 m_{b}}\left|\left(-i \vec{\nabla}+\frac{e}{c} \vec{A}\right) \psi(r)\right|^{2}\right\}+ \\
& \frac{1}{2} \int_{0}^{\beta} d \tau \int d^{2} r \int d^{2} r^{\prime} \psi^{\dagger} \psi(r) v\left(\vec{r}-\vec{r}^{\prime}\right) \psi^{\dagger} \psi\left(r^{\prime}\right)+ \\
& i \int_{0}^{\beta} d \tau \int d^{2} r \lambda(r)\left\{\frac{1}{4 m_{b}}\left[\psi^{\dagger}\left(-i \vec{\nabla}+\frac{e}{c} \vec{A}\right)^{2} \psi(r)+\left(\left(i \vec{\nabla}+\frac{e}{c} \vec{A}\right)^{2} \psi^{\dagger}\right) \psi(r)\right]-\frac{1}{2} \omega_{c} \psi^{\dagger} \psi(r)\right\} .
\end{aligned}
$$

In the above expression, $\mu$ is the chemical potential which fixes the LL filling fraction at $\nu . v(\vec{r})$ is a two-body interaction potential. In this paper we shall consider the Coulomb interaction explicitly in our calculation, where $v(\vec{r})=e^{2} / \epsilon r$, and $\epsilon$ is the dielectric constant. Other forms of interaction may be substituted straightforwardly into our calculations. The last term is a $\delta$-functional enforcing the constraint (11), where the kinetic energy term is symmetrized with respect to $\psi^{\dagger}$ and $\psi$. Notice that since the kinetic and potential energy term do not commute with each other, the constraint is intrinsically dynamical. Thus $\lambda(r) \equiv \lambda(\vec{r}, \tau)$ depends explicitly on the (imaginary) time $\tau$. This is different from that in the situation in, e.g., the slave boson approach for lattice fermions with infinite on-site repulsion in which the no-double-occupancy constraint may also be enforced by a $\delta$-functional [19]. The constraint equation there is a constant of motion hence $\lambda(r)=\lambda(\vec{r})$ is time independent. Because of the local nature of the auxiliary field $\lambda$, the kinetic energy in the last term may not be rearranged into the usual form $\frac{1}{2 m_{b}}\left|\left(-i \vec{\nabla}+\frac{e}{c} \vec{A}\right) \psi\right|^{2}$ through partial integration. In the spirit of composite fermion approach, we rewrite the action (3) in terms of the "transformed fermion fields", still denoted by $\psi^{\dagger}$ and $\psi$, such that

$$
\mathcal{Z}=\int \mathcal{D} \psi^{\dagger} \mathcal{D} \psi \mathcal{D} a_{\mu} \mathcal{D} \lambda e^{-S}
$$


with

$$
\begin{aligned}
& S=\int_{0}^{\beta} d \tau \int d^{2} r\left\{\psi^{\dagger}\left(\partial_{\tau}-i a_{0}-\mu\right) \psi+\frac{1}{2 m_{b}}\left|\left(-i \vec{\nabla}+\frac{e}{c} \vec{A}-\vec{a}\right) \psi\right|^{2}+\frac{i}{4 \pi \tilde{\phi}} \epsilon_{\mu \nu \lambda} a_{\mu} \partial_{\nu} a_{\lambda}\right\}+ \\
& \frac{1}{2} \int_{0}^{\beta} d \tau \int d^{2} r \int d^{2} r^{\prime} \psi^{\dagger}(r) \psi(r) v\left(\vec{r}-\vec{r}^{\prime}\right) \psi^{\dagger}\left(r^{\prime}\right) \psi\left(r^{\prime}\right)+ \\
& i \int_{0}^{\beta} d \tau \int d^{2} r \lambda(r)\left\{\frac{1}{4 m_{b}}\left[\psi^{\dagger}\left(-i \vec{\nabla}+\frac{e}{c} \vec{A}-\vec{a}\right)^{2} \psi(r)+\left(\left(i \vec{\nabla}+\frac{e}{c} \vec{A}-\vec{a}\right)^{2} \psi^{\dagger}\right) \psi(r)\right]-\frac{1}{2} \omega_{c} \psi^{\dagger}(r) \psi(r)\right\} .
\end{aligned}
$$

Here $\tilde{\phi}=2 p$, and $p$ is an integer. In the integral of $a_{\mu}$, a gauge fixing procedure is implicitly assumed. We attached even $(2 p)$ flux quanta per fermion through the well-known "ChernSimons term" with statistical gauge field $a_{\mu}$ : integrating out the 0 -component of $a_{\mu}$, we obtain

$$
|\vec{\nabla} \times \vec{a}|=2 \pi \tilde{\phi} \rho(r)
$$

where $\rho(r)=\psi^{\dagger} \psi(r)$ is a local density. While in the rest of the paper we will mainly focused on the ground state properties, we have formulated our theory within a finite temperature field theory formalism. Similar actions but without the constraint have been investigated in [20] in the context of anyon superconductivity and in [7] in a study of the FQHE at finite temperature.

Since the ground state of free fermions in a partially filled LL is highly degenerate, it is clearly not suitable for perturbative expansions. The above gauge transformation is motivated by the desire of finding a suitable mean-field theory which may provide a convenient starting point for approximations. Without the constraint and at zero $T$, this has been investigated by Lopez and Fradkin [1]: consider the homogeneous liquid saddle point solution for the Chern-Simons field $a_{\mu}=\bar{a}_{\mu}$, such that

$$
|\vec{\nabla} \times \overrightarrow{\bar{a}}|=2 \pi \tilde{\phi} \bar{\rho}, \quad \bar{a}_{0}=0
$$

where $\bar{\rho}$ is the average particle density. In this mean-field theory, the single-particle orbitals are described by the LLs determined by the effective magnetic field

$$
B_{e f f} \equiv\left|\vec{\nabla} \times \vec{A}_{e f f}\right| \equiv\left|\vec{\nabla} \times\left(\vec{A}-\frac{c}{e} \overrightarrow{\bar{a}}\right)\right|
$$


For the filling fraction $\nu$ such that $1 /(1 / \nu-2 p)=n$, an integer, $B_{\text {eff }}=\nu B / n$, the meanfield theory possesses an incompressible ground state described by $n$ filled (effective) LLs. The filling fraction $\nu$ which satisfies this condition is precisely the value where the FQHE arises. However, since this mean-field approximation for the Chern-Simons statistical gauge field $a_{\mu}$ does not depend on the nature of the underlying electronic state (other than the assumption that it is a homogeneous liquid), in the unconstrained theory this mapping of such a fractionally filled state onto an incompressible state also works (by which we mean that the mean-field solution exists and is stable against Gaussian fluctuations) for free electrons! This contradicts with the well known fact that the FQHE is a result of strong electron-electron correlations hence the mapping between the FQH states and the IQH states is sensible only when interactions are present. This serious deficiency of the unconstrained theory is reflected by the fact that the energy scale describing the FQH states in this theory is given the effective cyclotron energy $\hbar e B_{e f f} / m_{b} c$, which is proportional to the bare cyclotron energy $\hbar \omega_{c}[\mathbb{1}]$.

Now we consider the homogeneous mean-field solution with the constraint. The meanfield theory is obtained by substitutions $a_{\mu} \rightarrow \bar{a}_{\mu}, i \lambda(r) \rightarrow \bar{\lambda}$, where $\bar{a}_{\mu}$ is given by Eqn.((7) and $\bar{\lambda}$ is a constant, independent of $r$, such that $\partial \mathcal{F} / \partial \bar{\lambda}=0$ enforces the LLL constraint. In the free electron case, the ground state of the mean-field theory for the statistical field (『) is given by the configurations of electrons occupying the effective LLs subjected to the constraint

$$
\sum_{l} \hbar \bar{\omega}_{c}\left(l+\frac{1}{2}\right) n_{l}=\frac{1}{2} \hbar \omega_{c} N_{e}
$$

where $\bar{\omega}_{c}=e B_{e f f} / m_{b} c$ is the mean-field cyclotron frequency and $n_{l}$ is the occupancy of the l-th LL. Consider the example of $\nu=1 / 3$. In this case $\bar{\omega}_{c}=\frac{1}{3} \omega_{c}$, and the mean-field eigenspectrum is given by $\frac{\hbar \omega_{c}}{6}\left(l+\frac{1}{2}\right)$. As illustrated in Fig. 1, this ground state is highly degenerate as expected. Thus we see that there is indeed no resemblance of a fractionally filled state to the IQH state for free systems. Notice that in this (non-interacting) case, the mean-field value for the $\lambda$-field $i \lambda(r)=\bar{\lambda}$ is not a stationary point since the free energy is a 
linear function of $\bar{\lambda}$.

Consider now the interacting case. As shown in the Ref. [1,2], the treatment of interaction is equivalent to a treatment of the fluctuations in the gauge field $a_{\mu}$ because of the condition (6). Thus one may write the interaction term in the action (5) in terms of the statistical field $a_{\mu}$. Shift

$$
a_{\mu} \rightarrow \bar{a}_{\mu}+a_{\mu}, \quad i \lambda(r) \rightarrow \bar{\lambda}+i \lambda(r)
$$

so that $a_{\mu}$ and $\lambda$ represent fluctuations with respect to the saddle point solution, we write the action $S$ in the form $S=S_{0}+S_{1}$, with

$$
S_{0}=\int_{0}^{\beta} d \tau \int d^{2} r\left\{\psi^{\dagger}\left(\partial_{\tau}-\tilde{\mu}\right) \psi(r)+\frac{1}{2 m^{*}}\left|\left(-i \vec{\nabla}+\frac{e}{c} \vec{A}_{e f f}\right) \psi(r)\right|^{2}\right\}
$$

and

$$
\begin{aligned}
S_{1}= & \int_{0}^{\beta} d \tau \int d^{2} r\left\{\left(-i a_{0}-i \lambda(r) \frac{\omega_{c}}{2}\right) \rho(r)-\vec{j} \cdot \vec{a}(r)+\frac{1}{2 m^{*}} \vec{a} \cdot \vec{a} \rho(r)+\frac{i}{4 \pi \tilde{\phi}} \epsilon_{\mu \nu \lambda} a_{\mu} \partial_{\nu} a_{\lambda}\right\} \\
& +i \lambda(r) \frac{m^{*}}{m_{b}} h(r)-i \lambda(r) \frac{m^{*}}{m_{b}} \vec{j} \cdot \vec{a}(r)+i \lambda(r) \frac{1}{2 m_{b}} \vec{a} \cdot \vec{a} \rho(r) \\
& +\frac{1}{2} \int_{0}^{\beta} d \tau \int d^{2} r \int d^{2} r^{\prime} \frac{1}{(2 \pi \tilde{\phi})^{2}}[\vec{\nabla} \times \vec{a}(r)] v(\vec{r}-\vec{r})\left[\vec{\nabla} \times \vec{a}\left(r^{\prime}\right)\right]
\end{aligned}
$$

where

$$
\begin{aligned}
m^{*} & =m /(1+\bar{\lambda}), \\
\tilde{\mu} & =\mu+\frac{\bar{\lambda}}{2} \omega_{c},
\end{aligned}
$$

are renormalized mass and renormalized chemical potential respectively.

$$
\vec{j}(r)=-\frac{i}{2 m^{*}}\left(\psi^{\dagger} \vec{\nabla} \psi(r)-\left(\vec{\nabla} \psi^{\dagger}\right) \psi(r)\right)+\frac{1}{m^{*}} \frac{e}{c} \vec{A}_{e f f} \psi^{\dagger} \psi(r)
$$

is the mean-field current density and

$$
h(r)=\frac{1}{4 m^{*}}\left[\psi^{\dagger}\left(-i \vec{\nabla}+\frac{e}{c} \vec{A}_{e f f}\right)^{2} \psi(r)+\left(\left(i \vec{\nabla}+\frac{e}{c} \vec{A}_{e f f}\right)^{2} \psi^{\dagger}\right) \psi(r)\right]
$$

is the mean-field kinetic energy density. $\bar{\lambda}$ is determined by the extremal condition 


$$
\frac{\partial \mathcal{F}}{\partial \bar{\lambda}}=0
$$

Without $S_{1}$, the mean-field ground state is highly degenerate as discussed above. Following the "composite fermion" picture [3], it is nature to expect that a new stationary point solution, which describes $n$ filled (effective) LLs for the filling fraction $\nu=n /(1+2 p n)$, will emerge when the interaction is properly treated. However, it is a non-trivial task to determine which level of approximations would qualify to be the "proper treatment" of the interaction. In the present work, we will only examine the single loop quantum corrections described by a Gaussian theory.

As the very first step for constructing a loop expansion, one needs to decide which meanfield state should be taken. Based on the above discussion, we shall assume that fluctuations will shift the non-interacting mean-field ground state to a new one which is non-degenerate and is described by $n$ filled effective LLs for the filling fractions $\nu=n /(1+2 p n)$. With this new mean-field state, we calculate the (one) loop corrections and obtain the physical description of the corresponding FQH state. The correctness of our assumption will be determined later by a self-consistency requirement. As will be discussed below, the selfconsistency equation may be seen as the equation for the mass renormalization of composite fermions.

The fact that fluctuation corrections may result in a new stationary point state is well documented. For a charged superconductor, it is known that in certain situations [21] gauge field fluctuations may shift the order parameter and render the superconducting transition to first order, in contrast to the usual second order phase transition predicted by the GinzburgLandau theory without considering the fluctuations in the electric-magnetic field [22]. In quantum field theory, a similar case is also found in the scalar electrodynamics in four dimension [23]. In our case, the situation is slightly different since here we postulate the new mean-field solution first, compute the loop corrections with respect to this assumed mean-field solution, and verify the correctness (i.e., the existence and the stability of this new solution) self-consistently by solving the saddle point equation, which is now dressed by 
loop corrections. Our hope is that the one-loop theory will be sufficient to give the correct physics. An idea of similar spirit has been proposed previously by Weng in his treatment of doped quantum antiferromagnet [24].

If one ignores the fluctuations in $\lambda$, and considers only the Gaussian fluctuations in the statistical field $a_{\mu}$, one would obtain similar results as those of the unconstrained theory [1.7], but with a renormalized mass $m^{*}$. However, this simple minded approach violates Kohn's theorem 25]. Thus it is essential to incorporate the fluctuations in $\lambda$, treat them at the same footing as those in the statistical field $a_{\mu}$.

As noted in [2], in a theory without constraint, one may utilize the continuity equation to simplify the calculation of the response kernel through some special choice of gauge. For a system of electrons constrained to the LLL, however, it is in general not possible to apply such a procedure since in this case the current defined in the usual manner does not satisfy the continuity equation [12, 13]. Thus we shall use the gauge invariant formalism of [1]. Denote $\left(a_{\mu} i \lambda\right) \equiv\left(i a_{0} a_{x} a_{y} i \lambda\right)$, the Gaussian action may be expressed as

$$
S_{\text {Gaussian }}\left(a_{\mu} ; i \lambda\right)=\frac{1}{2} \sum_{\vec{q}, i \omega_{n}}\left(a_{\mu}\left(-\vec{q},-i \omega_{n}\right) \quad i \lambda\left(-\vec{q},-i \omega_{n}\right)\right) D^{-1}\left(\vec{q}, i \omega_{n}\right)\left(\begin{array}{c}
a_{\mu}\left(\vec{q}, i \omega_{n}\right) \\
i \lambda\left(\vec{q}, i \omega_{n}\right)
\end{array}\right)
$$

with the kernel

$$
D^{-1}\left(\vec{q}, i \omega_{n}\right)=U\left(\vec{q}, i \omega_{n}\right)+\Pi^{0}\left(\vec{q}, i \omega_{n}\right)
$$

Here $U$ is the tree diagram contribution

$$
U\left(\vec{q}, i \omega_{n}\right)=\frac{1}{2 \pi \tilde{\phi}}\left(\begin{array}{cccc}
0 & -i q_{y} & i q_{x} & 0 \\
i q_{y} & \tilde{\omega}_{c} \frac{\mu_{s}}{2 p} \frac{q_{y}^{2}}{q^{2}} \tilde{l}_{0} q & i\left(i \omega_{n}\right)-\tilde{\omega}_{c} \frac{\mu_{s}}{2 p} \frac{q_{x} q_{y}}{q^{2}} \tilde{l}_{0} q & 0 \\
-i q_{x} & -i\left(i \omega_{n}\right)-\tilde{\omega}_{c} \frac{\mu_{s}}{2 p} \frac{q_{x} q_{y}}{q^{2}} \tilde{l}_{0} q & \tilde{\omega}_{c} \frac{\mu_{s}}{2 p} \frac{q_{x}^{2}}{q^{2}} \tilde{l}_{0} q & 0 \\
0 & 0 & 0 & 0
\end{array}\right)
$$

and $\Pi^{0}\left(\vec{q}, i \omega_{n}\right)$ gives the one-loop contribution (for $i \omega_{n} \neq 0$ ),

$\Pi^{0}\left(\vec{q}, i \omega_{n}\right)=$ 
$\frac{\tilde{n}}{2 \pi \hat{\phi}}\left(\begin{array}{cccc}\frac{q^{2}}{\tilde{\omega}_{c}} \Sigma_{0} & \frac{i \omega_{n}}{\tilde{\omega}_{c}} q_{x} \Sigma_{0}+i q_{y} \Sigma_{1} & -i q_{x} \Sigma_{1}+\frac{i \omega_{n}}{\tilde{\omega}_{c}} q_{y} \Sigma_{0} & \frac{\omega_{c}}{2} \frac{q^{2}}{\tilde{\omega}_{c}} \Sigma_{4} \\ \frac{i \omega_{n}}{\tilde{\omega}_{c}} q_{x} \Sigma_{0}-i q_{y} \Sigma_{1} & \tilde{\omega}_{c}\left(\left(\frac{i \omega_{n}}{\tilde{\omega}_{c}}\right)^{2} \Sigma_{0}+\frac{q_{y}^{2}}{q^{2}} \Sigma_{3}\right) & -i\left(i \omega_{n}\right) \Sigma_{1}-\tilde{\omega}_{c} \frac{q_{x} q_{y}}{q^{2}} \Sigma_{3} & \frac{\omega_{c}}{2}\left(\frac{i \omega_{n}}{\tilde{\omega}_{c}} q_{x} \Sigma_{4}-i q_{y} \Sigma_{5}\right) \\ i q_{x} \Sigma_{1}+\frac{i \omega_{n}}{\tilde{\omega}_{c}} q_{y} \Sigma_{0} & i\left(i \omega_{n}\right) \Sigma_{1}-\tilde{\omega}_{c} \frac{q_{x} q_{y}}{q^{2}} \Sigma_{3} & \tilde{\omega}_{c}\left(\left(\frac{i \omega_{n}}{\tilde{\omega}_{c}}\right)^{2} \Sigma_{0}+\frac{q_{x}^{2}}{q^{2}} \Sigma_{3}\right) & \frac{\omega_{c}}{2}\left(i q_{x} \Sigma_{5}+\frac{i \omega_{n}}{\tilde{\omega}_{c}} q_{y} \Sigma_{4}\right) \\ \frac{\omega_{c}}{2} \frac{q^{2}}{\tilde{\omega}_{c}} \Sigma_{4} & \frac{\omega_{c}}{2}\left(\frac{i \omega_{n}}{\tilde{\omega}_{c}} q_{x} \Sigma_{4}+i q_{y} \Sigma_{5}\right) & \frac{\omega_{c}}{2}\left(-i q_{x} \Sigma_{5}+\frac{i \omega_{n}}{\tilde{\omega}_{c}} q_{y} \Sigma_{4}\right) & \left(\frac{\omega_{c}}{2}\right)^{2} \frac{q^{2}}{\tilde{\omega}_{c}} \Sigma_{6}\end{array}\right)$,

with $\tilde{n}=2 p n$ and

$$
\begin{aligned}
\Sigma_{j}= & \frac{e^{-x}}{n} \sum_{m<l} \frac{l-m}{\left(\frac{i \omega_{n}}{\tilde{\omega}_{c}}\right)^{2}-(l-m)^{2}} \frac{m !}{l !} x^{l-m-1}\left\{f\left(\epsilon_{m}-\tilde{\mu}\right)-f\left(\epsilon_{l}-\tilde{\mu}\right)\right\} \\
& {\left[L_{m}^{l-m}(x)\right]^{2-j}\left[(l-m-x) L_{m}^{l-m}(x)+2 x \frac{d L_{m}^{l-m}(x)}{d x}\right]^{j} }
\end{aligned}
$$

for $\mathrm{j}=0,1,2$ [26]; and

$$
\begin{gathered}
\Sigma_{3}=\frac{e^{-x}}{n} \sum_{m<l} \frac{l-m}{\left(\frac{i \omega_{n}}{\tilde{\omega}_{c}}\right)^{2}-(l-m)^{2}} \frac{m !}{l !} x^{l-m}\left\{f\left(\epsilon_{m}-\tilde{\mu}\right)-f\left(\epsilon_{l}-\tilde{\mu}\right)\right\} \\
{\left[2 \frac{d L_{m}^{l-m}(x)}{d x}-L_{m}^{l-m}(x)\right]\left[(2(l-m)-x) L_{m}^{l-m}(x)+2 x \frac{d L_{m}^{l-m}(x)}{d x}\right],} \\
\Sigma_{4}=\frac{e^{-x}}{n} \sum_{m<l} \frac{l-m}{\left(\frac{i \omega_{n}}{\tilde{\omega}_{c}}\right)^{2}-(l-m)^{2}} \frac{m !}{l !} x^{l-m-1}\left\{f\left(\epsilon_{m}-\tilde{\mu}\right)-f\left(\epsilon_{l}-\tilde{\mu}\right)\right\}\left(1-\frac{l+m+1}{1+\tilde{n}}\right)\left[L_{m}^{l-m}(x)\right]^{2}, \\
\Sigma_{5}=\frac{e^{-x}}{n} \sum_{m<l} \frac{l-m}{\left(\frac{i \omega_{n}}{\tilde{\omega}_{c}}\right)^{2}-(l-m)^{2}} \frac{m !}{l !} x^{l-m-1}\left\{f\left(\epsilon_{m}-\tilde{\mu}\right)-f\left(\epsilon_{l}-\tilde{\mu}\right)\right\} \\
\left(1-\frac{l+m+1}{1+\tilde{n}}\right) L_{m}^{l-m}(x)\left[(l-m-x) L_{m}^{l-m}(x)+2 x \frac{d L_{m}^{l-m}(x)}{d x}\right], \\
\Sigma_{6}=\frac{e^{-x}}{n} \sum_{m<l} \frac{l-m}{\left(\frac{i \omega_{n}}{\tilde{\omega}_{c}}\right)^{2}-(l-m)^{2}} \frac{m !}{l !} x^{l-m-1}\left\{f\left(\epsilon_{m}-\tilde{\mu}\right)-f\left(\epsilon_{l}-\tilde{\mu}\right)\right\}\left(1-\frac{l+m+1}{1+\tilde{n}}\right)^{2}\left[L_{m}^{l-m}(x)\right]^{2} .
\end{gathered}
$$

In the above equations, $i \omega_{n}$ is the Matsubara frequency, $L_{m}^{l}$ is the Laguerre polynomial, $x=\left(\tilde{l}_{0} q\right)^{2} / 2$, and $\tilde{l}_{0}=\sqrt{c / e B_{e f f}}, \tilde{\omega}_{c}=e B_{e f f} / m^{*} c$, are the effective magnetic length and the effective cyclotron frequency respectively. $\mu_{s}$ is the ratio of $\tilde{l}_{0}$ to the effective Bohr radius $a_{0}=\epsilon / m^{*} e^{2}$. The chemical potential $\mu$ is renormalized by $\bar{\lambda}$ according to (14) such that there are precisely $n$ LL are filled 20]:

$$
n=\sum_{l} f\left(\epsilon_{l}-\tilde{\mu}\right), \quad f\left(\epsilon_{l}-\tilde{\mu}\right)=\frac{1}{e^{\beta\left(\epsilon_{l}-\tilde{\mu}\right)}+1}
$$


where $\epsilon_{l}=\tilde{\omega}_{c}\left(l+\frac{1}{2}\right)$. For the case $i \omega_{n}=0$, the one-loop contribution is given by

$$
\Pi^{0}\left(\vec{q}, i \omega_{n}=0\right)=\frac{\tilde{n}}{2 \pi \tilde{\phi}}\left(\begin{array}{cccc}
\frac{q^{2}}{\tilde{\omega}_{c}} \Sigma_{0}^{0} & i q_{y} \Sigma_{1}^{0} & -i q_{x} \Sigma_{1}^{0} & \frac{\omega_{c}}{2} \frac{q^{2}}{\tilde{\omega}_{c}} \Sigma_{4}^{0} \\
-i q_{y} \Sigma_{1}^{0} & \tilde{\omega}_{c} \frac{q_{y}^{2}}{q^{2}} \Sigma_{3}^{0} & -\tilde{\omega}_{c} \frac{q_{x} q_{y}}{q^{2}} \Sigma_{3}^{0} & -i \frac{\omega_{c}}{2} q_{y} \Sigma_{5}^{0} \\
i q_{x} \Sigma_{1}^{0} & -\tilde{\omega}_{c} \frac{q_{x} q_{y}}{q^{2}} \Sigma_{3}^{0} & \tilde{\omega}_{c} \frac{q_{x}^{2}}{q^{2}} \Sigma_{3}^{0} & i \frac{\omega_{c}}{2} q_{x} \Sigma_{5}^{0} \\
\frac{\omega_{c}}{2} \frac{q^{2}}{\tilde{\omega}_{c}} \Sigma_{4}^{0} & i \frac{\omega_{c}}{2} q_{y} \Sigma_{5}^{0} & -i \frac{\omega_{c}}{2} q_{x} \Sigma_{5}^{0} & \left(\frac{\omega_{c}}{2}\right)^{2} \frac{q^{2}}{\tilde{\omega}_{c}} \Sigma_{6}^{0}
\end{array}\right),
$$

where

$$
\Sigma_{j}^{0}(\vec{q})=\Sigma_{j}(\vec{q}, 0)-\frac{\beta \tilde{\omega}_{c}}{2} \frac{e^{-x}}{n} x^{j-1} \sum_{m} e^{\left(\epsilon_{m}-\tilde{\mu}\right)} f^{2}\left(\epsilon_{m}-\tilde{\mu}\right)\left[L_{m}^{0}(x)\right]^{2-j}\left[\left(L_{m}^{0}(x)-2 L_{m}^{1}(x)\right]^{j}\right.
$$

for $\mathrm{j}=0,1,2$;

$$
\begin{gathered}
\Sigma_{3}^{0}(\vec{q})=\Sigma_{2}^{0}(\vec{q}) \\
\Sigma_{4}^{0}(\vec{q})=\Sigma_{4}(\vec{q}, 0)-\frac{\beta \tilde{\omega}_{c}}{2} \frac{e^{-x}}{n x} \sum_{m}\left(1-\frac{2 m+1}{1+\tilde{n}}\right) e^{\left(\epsilon_{m}-\tilde{\mu}\right)} f^{2}\left(\epsilon_{m}-\tilde{\mu}\right)\left[L_{m}^{0}(x)\right]^{2} \\
\Sigma_{5}^{0}(\vec{q})=\Sigma_{5}(\vec{q}, 0)-\frac{\beta \tilde{\omega}_{c}}{2} \frac{e^{-x}}{n} \sum_{m}\left(1-\frac{2 m+1}{1+\tilde{n}}\right) e^{\left(\epsilon_{m}-\tilde{\mu}\right)} f^{2}\left(\epsilon_{m}-\tilde{\mu}\right) L_{m}^{0}(x)\left[L_{m}^{0}(x)-2 L_{m}^{1}(x)\right], \\
\Sigma_{6}^{0}(\vec{q})=\Sigma_{6}(\vec{q}, 0)-\frac{\beta \tilde{\omega}_{c}}{2} \frac{e^{-x}}{n x} \sum_{m}\left[1-\frac{2 m+1}{1+\tilde{n}}\right]^{2} e^{\left(\epsilon_{m}-\tilde{\mu}\right)} f^{2}\left(\epsilon_{m}-\tilde{\mu}\right)\left[L_{m}^{0}(x)\right]^{2}
\end{gathered}
$$

The computation for $\Pi^{0}$ is detailed in the appendix. Notice that because there are no tree diagrams involving the auxiliary field $i \lambda(r)$, the matrix $U$ possesses no inverse. The free energy is obtained by completing the functional integral (with the standard gauge fixing procedure) of $a_{\mu}$ and $\lambda$ in the partition function:

$$
\mathcal{F}=\mathcal{F}_{0}+\frac{1}{2 \beta} \sum_{\vec{q}, i \omega_{n}} \ln \operatorname{det} D^{-1}\left(\vec{q}, i \omega_{n}\right),
$$

where $\mathcal{F}_{0}$ is the mean-field contribution to the free energy and the determinant of $D, \operatorname{det} D$, is given by, apart from a constant (in $\bar{\lambda}$ ) factor (see [26]),

$$
\begin{aligned}
\operatorname{det} D= & \tilde{n}\left(\Sigma_{0} \Sigma_{6}-\Sigma_{4}^{2}\right) \frac{\mu_{s}}{2 p} \tilde{l}_{0} q+\tilde{n}^{2}\left(\Sigma_{0} \Sigma_{6}-\Sigma_{4}^{2}\right)\left[\left(\frac{i \omega_{n}}{\tilde{\omega}_{c}}\right)^{2} \Sigma_{0}+\Sigma_{3}\right] \\
& -\Sigma_{6}\left(1-\tilde{n} \Sigma_{1}\right)^{2}-\tilde{n}^{2} \Sigma_{0} \Sigma_{5}^{2}-2 \tilde{n} \Sigma_{4} \Sigma_{5}\left(1-\tilde{n} \Sigma_{1}\right)
\end{aligned}
$$


The saddle point value $\bar{\lambda}$ is given by the extremal condition (17) which, in the present case of one-loop expansion, can be written as

$$
\frac{\partial \mathcal{F}_{0}}{\partial \bar{\lambda}}+\frac{1}{2 \beta} \sum_{\vec{q}, i \omega_{n}} \frac{1}{\operatorname{det} D\left(\vec{q}, i \omega_{n}\right)} \frac{\partial \operatorname{det} D\left(\vec{q}, i \omega_{n}\right)}{\partial \bar{\lambda}}=0 .
$$

If one lets $\mu_{s}=0$ in (35), which corresponds to that for a non-interacting system with Gaussian fluctuation corrections (in both $a_{\mu}$ and $\lambda$ ), one would easily see that $\mathcal{F}$ is linear in $\bar{\lambda}$ and therefore there is no saddle point. Because of the interaction, the free energy is no more a linear function of $\bar{\lambda}$, hence one may expect a non-trivial solution. Thus, after taking a mean-field state of integer-filled pseudo LLs (which is not a legitimate state for non-interacting electrons but is assumed to emerge as a valid one when the interaction is switched on) as our starting point for the loop expansion, our assumption is checked selfconsistently by finding solutions for (36), in which the free energy is calculated with the one-loop fluctuation corrections.

From the expression of $S_{0}$, Eqn.(11), it is tempting to interpret $m^{*} \equiv m /(1+\bar{\lambda})$ as an "effective mass" of the composite fermions. (Notice that the stability of the mean field solution requires that $1+\bar{\lambda}>0$.) Thus the above equation (36) may be seen as the mass renormalization equation for composite fermions. While nominally this definition of the effective mass differs from the usual one [2,18], it too is a result of electron-electron interactions and gauge field fluctuations. And we shall see later, $m^{*}$ also measures the energy gap in the excitation spectrum. Incorporating higher order loop expansions will in general further shift $\bar{\lambda}$, giving additional corrections to the effective mass.

\section{ELECTRO-MAGNETIC RESPONSE}

The electro-magnetic response for a system constrained to the LLL is a quite subtle

problem. Consider fluctuations in $A_{\mu}$ such that $A_{\mu} \rightarrow A_{\mu}+\delta A_{\mu}$. It is tempting to write the action describing the system with this additional fluctuating electric-magnetic field as

$S=\int_{0}^{\beta} d \tau \int d^{2} r\left\{\psi^{\dagger}\left(\partial_{\tau}+i \delta A_{0}-\mu\right) \psi(r)+\frac{1}{2 m_{b}}\left|\left(-i \vec{\nabla}+\frac{e}{c}(\vec{A}+\delta \vec{A})\right) \psi(r)\right|^{2}\right\}+$ 


$$
\begin{aligned}
& \frac{1}{2} \int_{0}^{\beta} d \tau \int d^{2} r \int d^{2} r^{\prime} \psi^{\dagger} \psi(r) v\left(\vec{r}-\vec{r}^{\prime}\right) \psi^{\dagger} \psi\left(r^{\prime}\right)+ \\
& i \int_{0}^{\beta} d \tau \int d^{2} r \lambda(r)\left\{\frac{1}{4 m_{b}}\left[\psi^{\dagger}\left(-i \vec{\nabla}+\frac{e}{c} \vec{A}\right)^{2} \psi(r)+\left(\left(i \vec{\nabla}+\frac{e}{c} \vec{A}\right)^{2} \psi^{\dagger}\right) \psi(r)\right]-\frac{1}{2} \omega_{c} \psi^{\dagger} \psi(r)\right\} .
\end{aligned}
$$

Notice that the term involving the $\lambda$-field is independent of $\delta A_{\mu}$. One would expect that such an action describes the electro-magnetic response of the system to the perturbation $\delta A_{\mu}$ while it is confined to the Hilbert space of the LLL of the unperturbed system, consistent with the "standard approach" using trial wavefunctions [4]. However, as it stands this action is not invariant under the gauge transformation

$$
\delta A_{\mu} \rightarrow \delta A_{\mu}+\partial_{\mu} f
$$

Because one does not have the usual "minimal coupling" for the external perturbation $\delta A_{\mu}$, the current density cannot be simply obtained from the derivative

$$
j_{\mu}=\frac{\partial S}{\partial A_{\mu}}
$$

On the other hand, the action (3) (or equivalently (5)) clearly preserves the global $U(1)$ symmetry of the unconstrained theory, one may thus still define a Noether current density accordingly. As usual, consider a phase change in the fermion field such that

$$
\psi \rightarrow e^{i \epsilon} \psi, \quad \psi^{\dagger} \rightarrow \psi^{\dagger} e^{-i \epsilon}
$$

Invariance of the action with respect to an arbitrary infinitesimal change of $\epsilon$ lead to a conserved current density $j_{\mu}^{\lambda}$, which in terms of the Chern-Simons transformed fields is given by

$$
\begin{aligned}
j_{0}^{\lambda} & =\psi^{\dagger} \psi(r), \\
\vec{j}^{\lambda} & =\vec{j}(r)-\frac{\vec{a}}{m^{*}} \psi^{\dagger} \psi(r)+i \lambda(r)\left\{-\frac{i}{2 m_{b}}\left[\psi^{\dagger} \vec{\nabla} \psi(r)-\left(\vec{\nabla} \psi^{\dagger}\right) \psi(r)\right]+\frac{1}{m_{b}}\left(\frac{e}{c} \vec{A}_{e f f}-\vec{a}\right) \psi^{\dagger} \psi(r)\right\},
\end{aligned}
$$

where $\vec{j}(r)$ is the mean-field current density defined in (15). $j_{\mu}^{\lambda}$ is, of course, not the physical current density measured in experiments, which must be obtained by integrating out the auxiliary field $\lambda$ [13]. 
To study the electro-magnetic response, we shall consider a simpler case where the external fluctuating field is in the time component only, i.e., $\delta A_{\mu}=\left(i \delta A_{0}, 0,0\right)$, so that it couples to the system according to (3) with the substitution $A_{\mu} \rightarrow A_{\mu}+\delta A_{\mu}$. The action is then invariant under the gauge transformation $A_{\mu}+\delta A_{\mu} \rightarrow A_{\mu}+\delta A_{\mu}+\partial_{\mu} f$, and the system under such a perturbation $\delta A_{\mu}$ is still constrained to the (original) LLL. Using the standard Faddeev-Popov procedure (where the gauge fixing term can be chosen as, e.g., $\left.\frac{1}{2 \alpha}\left(\partial_{\mu} a_{\mu}\right)^{2}\right)$, one may integrate out the statistical field $a_{\mu}$ and arrives an effective action for such a $\delta A_{\mu}$. After some algebra, one obtains

$$
\tilde{S}_{\text {Gaussian }}\left(\delta A_{0}\right)=\frac{1}{2} \sum_{\vec{q}, i \omega_{n}} i \delta A_{0}\left(-\vec{q},-i \omega_{n}\right) K_{00}\left(\vec{q}, i \omega_{n}\right) i \delta A_{0}\left(\vec{q}, i \omega_{n}\right)
$$

One may interpret $K_{00}$ as the density-density correlation function [13

$$
\rho\left(\vec{q}, i \omega_{n}\right)=\frac{\delta \tilde{S}}{i \delta A_{0}}=K_{00}\left(\vec{q}, i \omega_{n}\right) i \delta A_{0}\left(\vec{q}, i \omega_{n}\right)
$$

For $i \omega \neq 0$,

$$
K_{00}\left(\vec{q}, i \omega_{n}\right)=\frac{\tilde{n}}{2 \pi \tilde{\phi} \operatorname{det} D\left(\vec{q}, i \omega_{n}\right)} \frac{q^{2}}{\tilde{\omega}_{c}}\left(\Sigma_{4}^{2}-\Sigma_{0} \Sigma_{6}\right)
$$

The linear response is obtained by the standard procedure $i \omega_{n} \rightarrow \omega+i \eta$. Thus one may find the collective excitation spectrum by locating the poles in $K_{00}$. Notice that if one applies a similar procedure to a system without the constraint, one would obtain the same response kernel $K_{00}$ as that with an arbitrary $\delta A_{\mu}$. Hence it is reasonable to expect that $K_{00}$ gives the correct density-density correlation, and is sufficient for the purpose of studying physics other than transport properties.

The compressibility is obtained from

$$
\kappa=\lim _{q \rightarrow 0} K_{00}\left(\vec{q}, i \omega_{n}=0\right)=\lim _{q \rightarrow 0} \frac{\tilde{n}}{2 \pi \tilde{\phi} \operatorname{det} D^{0}(\vec{q})} \frac{q^{2}}{\tilde{\omega}_{c}}\left(\left(\Sigma_{4}^{0}\right)^{2}-\Sigma_{0}^{0} \Sigma_{6}^{0}\right),
$$

where $D^{0}(\vec{q})$ is obtained from (35) with the substitution $\Sigma_{j} \rightarrow \Sigma_{j}^{0}$. At $T=0, \kappa=0$, the system is incompressible. As $T$ is raised from zero, the compressibility acquires an exponential correction as expected. 


\section{DISCUSSIONS AND CONCLUDING REMARKS}

The mass renormalization equation (36) can in principle be solved numerically, which is quite complicated due to the large (infinite) number of singularities in the integrand. We shall not discuss it the present work. Instead, we will offer a conjecture on a solution based on physical considerations. Since the physical energy scale is given by the electron-electron interaction, we have

$$
\frac{e B}{m^{*} c} \propto \frac{e^{2}}{\epsilon l_{0}}
$$

when $v(\vec{r})$ is the Coulomb interaction. Here $l_{0}$ is the bare magnetic length, $l_{0}=\sqrt{\frac{\nu}{n}} \tilde{l}_{0}$. We may in this case write $(\hbar=1)$

$$
\bar{\lambda}=-1+\gamma \frac{e^{2} / \epsilon l_{0}}{\omega_{c}}
$$

where $\gamma$ is a numerical factor (to be determined from (36])).

A strict constraint on any theory of electrons in magnetic field is the celebrated Kohn's theorem, which states that in an impurity free system the total oscillation strength $K_{00}$ is saturated by the cyclotron mode (to the order $q^{2}$ ), regardless the details of interactions. Since in our theory the system is constrained to the LLL, the cyclotron mode is pushed up to infinite. Kohn's theorem then requires that all the modes in our theory must be weighted with some higher power then $q^{2}$ as $q \rightarrow 0$. To ensure the one-loop expansion adopted here to be sensible, we must demonstrate that Kohn's theorem is indeed satisfied within our Gaussian theory. Below we show this explicitly for the case $T=0$.

As $\beta \rightarrow \infty$, we have

$\Sigma_{j}=\frac{e^{-x}}{n} \sum_{m=0}^{n-1} \sum_{l=n}^{\infty} \frac{l-m}{\left(\frac{\omega}{\tilde{\omega}_{c}}\right)^{2}-(l-m)^{2}} \frac{m !}{l !} x^{l-m-1}\left[L_{m}^{l-m}(x)\right]^{2-j}\left[(l-m-x) L_{m}^{l-m}(x)+2 x \frac{d L_{m}^{l-m}(x)}{d x}\right]^{j}$

for $\mathrm{j}=0,1,2$; and

$$
\begin{aligned}
\Sigma_{3}= & \frac{e^{-x}}{n} \sum_{m=0}^{n-1} \sum_{l=n}^{\infty} \frac{l-m}{\left(\frac{\omega}{\tilde{\omega}_{c}}\right)^{2}-(l-m)^{2}} \frac{m !}{l !} x^{l-m} \\
& {\left[2 \frac{d L_{m}^{l-m}(x)}{d x}-L_{m}^{l-m}(x)\right]\left[(2(l-m)-x) L_{m}^{l-m}(x)+2 x \frac{d L_{m}^{l-m}(x)}{d x}\right], }
\end{aligned}
$$




$$
\begin{aligned}
\Sigma_{4}= & \frac{e^{-x}}{n} \sum_{m=0}^{n-1} \sum_{l=n}^{\infty} \frac{l-m}{\left(\frac{\omega}{\tilde{\omega}_{c}}\right)^{2}-(l-m)^{2}} \frac{m !}{l !} x^{l-m-1}\left(1-\frac{l+m+1}{1+\tilde{n}}\right)\left[L_{m}^{l-m}(x)\right]^{2}, \\
\Sigma_{5}= & \frac{e^{-x}}{n} \sum_{m=0}^{n-1} \sum_{l=n}^{\infty} \frac{l-m}{\left(\frac{\omega}{\tilde{\omega}_{c}}\right)^{2}-(l-m)^{2}} \frac{m !}{l !} x^{l-m-1} \\
& \left(1-\frac{l+m+1}{1+\tilde{n}}\right) L_{m}^{l-m}(x)\left[(l-m-x) L_{m}^{l-m}(x)+2 x \frac{d L_{m}^{l-m}(x)}{d x}\right], \\
\Sigma_{6}= & \frac{e^{-x}}{n} \sum_{m=0}^{n-1} \sum_{l=n}^{\infty} \frac{l-m}{\left(\frac{\omega}{\tilde{\omega}_{c}}\right)^{2}-(l-m)^{2}} \frac{m !}{l !} x^{l-m-1}\left(1-\frac{l+m+1}{1+\tilde{n}}\right)^{2}\left[L_{m}^{l-m}(x)\right]^{2},
\end{aligned}
$$

For $j=0,1,2,3$, they are just the results obtained previously for the unconstrained theory at $T=0$ [1]. As $q \rightarrow 0, \Sigma_{j}$ may be expanded to the order $x=\tilde{l}_{0}^{2} q^{2} / 2$ :

$$
\begin{aligned}
& \Sigma_{0}=\frac{1}{\left(\frac{\omega}{\tilde{\omega}_{c}}\right)^{2}-1}+\frac{3 n}{\left(\left(\frac{\omega}{\tilde{\omega}_{c}}\right)^{2}-1\right)\left(\left(\frac{\omega}{\tilde{\omega}_{c}}\right)^{2}-4\right)} x+\ldots \\
& \Sigma_{1}=\frac{1}{\left(\frac{\omega}{\tilde{\omega}_{c}}\right)^{2}-1}+\frac{6 n}{\left(\left(\frac{\omega}{\tilde{\omega}_{c}}\right)^{2}-1\right)\left(\left(\frac{\omega}{\tilde{\omega}_{c}}\right)^{2}-4\right)} x+\ldots \\
& \Sigma_{2}=\frac{1}{\left(\frac{\omega}{\tilde{\omega}_{c}}\right)^{2}-1}+\frac{n\left(\left(\frac{\omega}{\tilde{\omega}_{c}}\right)^{2}+8\right)}{\left(\left(\frac{\omega}{\tilde{\omega}_{c}}\right)^{2}-1\right)\left(\left(\frac{\omega}{\tilde{\omega}_{c}}\right)^{2}-4\right)} x+\ldots \\
& \Sigma_{3}=-\frac{2 n}{\left(\frac{\omega}{\tilde{\omega}_{c}}\right)^{2}-1} x+\ldots \\
& \Sigma_{4}=\frac{1}{\left(\frac{\omega}{\tilde{\omega}_{c}}\right)^{2}-1} \frac{1+\tilde{n}-2 n}{1+\tilde{n}}+\frac{1-\left(\frac{\omega}{\tilde{\omega}_{c}}\right)^{2}+3 n(1+\tilde{n}-2 n)}{\left(\left(\frac{\omega}{\tilde{\omega}_{c}}\right)^{2}-1\right)\left(\left(\frac{\omega}{\tilde{\omega}_{c}}\right)^{2}-4\right)(1+\tilde{n})} x+\ldots \\
& \Sigma_{5}=\frac{1}{\left(\frac{\omega}{\tilde{\omega}_{c}}\right)^{2}-1} \frac{1+\tilde{n}-2 n}{1+\tilde{n}}+2 \frac{1-\left(\frac{\omega}{\tilde{\omega}_{c}}\right)^{2}+3 n(1+\tilde{n}-2 n)}{\left(\left(\frac{\omega}{\tilde{\omega}_{c}}\right)^{2}-1\right)\left(\left(\frac{\omega}{\tilde{\omega}_{c}}\right)^{2}-4\right)(1+\tilde{n})} x+\ldots \\
& \Sigma_{6}=\frac{1}{\left(\frac{\omega}{\tilde{\omega}_{c}}\right)^{2}-1}\left(\frac{1+\tilde{n}-2 n}{1+\tilde{n}}\right)^{2}+\frac{(5 n-2 \tilde{n}-2)\left(\left(\frac{\omega}{\tilde{\omega}_{c}}\right)^{2}-1\right)+3 n(1+\tilde{n}-2 n)^{2}}{\left(\left(\frac{\omega}{\tilde{\omega}_{c}}\right)^{2}-1\right)\left(\left(\frac{\omega}{\tilde{\omega}_{c}}\right)^{2}-4\right)(1+\tilde{n})^{2}} x+\ldots
\end{aligned}
$$

Substitute these results to (44), we obtain the small $q$ behavior of the density-density response kernel 


$$
K_{00}(q, \omega)=\frac{\tilde{n}}{4 \pi \tilde{\phi}} \frac{q^{4} \tilde{l}_{0}^{2}}{\tilde{\omega}_{c}} \frac{\tilde{n}}{(1+\tilde{n}-2 n)^{2}} \frac{1}{\left(\frac{\omega}{\tilde{\omega}_{c}}\right)^{2}-4}+\ldots
$$

Thus one finds that the weight of the poles in $K_{00}$ vanishes according to $q^{4}$, satisfying Kohn's theorem. This feature of our theory is non-trivial and is quite encouraging, indicates strongly that this formalism may have well captured the correct low energy physics of the FQHE. Notice that the situation here is quite different from that in the unconstrained theory [1] in which the cyclotron mode is explicitly present and sets the energy scale of all the low energy excitations. Incidentally, (60) also shows that the energy gap at $q=0($ at $T=0)$ is $2 \tilde{\omega}_{c}=\left(\frac{2 \gamma}{1+\tilde{n}}\right) \frac{e^{2}}{\epsilon l_{0}}$. From our knowledge about the collective energy gap obtained through numerical works [4, [6, 16], we may estimate that $\gamma \approx 0.3$ for $\nu=1 / 3$.

One may also calculate the dispersion of the low energy collective modes at finite $q$ by finding the poles of $K_{00}$. It depends on the details (such as the range and strength) of the electron-electron interaction, as well as $\bar{\lambda}$. The numerical calculation of the full dispersion of the collective modes is straightforward once the value of $\bar{\lambda}$ is known. However, it is not clear that the above estimate for $\gamma$ is correct within the one-loop expansion. It is well possible (especially in view of the dynamical nature of the $\lambda$-field) that one needs corrections beyond the one-loop level to give a reasonable value for $\gamma$. These issues, of course, can only be clarified by actually solving the stationary point equation (36), which we hope to be done in the near future. In the absence of the solution for the mass renormalization equation (36), we shall not address the quantitative questions such as the dispersion of the collective modes in the present work.

To summarize, we present in this paper a fermion Chern-Simons field theory describing two-dimensional electrons in the LLL. The constraint is realized through a $\delta$-functional represented by an auxiliary field $\lambda$ which fixes the kinetic energy of electrons at $\frac{1}{2} \hbar \omega_{c}$. In a mean-field approximation, the ground state is highly degenerate for non-interacting systems when $\nu$ is not an integer. Assuming a new non-degenerate mean field ground state at $\nu=n /(2 p n+1)$ (which is described by $n$ filled effective LLs) for interacting electrons, we perform the one-loop expansion around this mean-field state and subject our assumption 
to an self-consistency requirement. The resulting dressed stationary point equation for $\bar{\lambda}$ is then physically interpreted as the mass renormalization equation for the composite fermions. An important issue which needs to be investigated in the future is to numerically solve the renormalization equation to provide an answer for the the energy gap of a FQH state in the thermodynamical limit.

\section{Acknowledgement}

The author thanks G.S. Canright, M. Ma and especially J.K. Jain for many stimulating discussions, and W. Chen, S.M. Girvin, A.H. MacDonald, J. Quinn, Z.B. Su and Y.S. Wu for helpful comments. This work was supported by the National Science Foundation under Grant No. DMR-9413057, and by the U.S. Department of Energy through Contract No. DE-AC05-84OR21400 administered by Martin Marietta Energy Systems Inc..

Appendix

In this appendix, we give some details of calculations of the matrix elements in $\Pi^{0}$. At zero $T$ and without the constraint, this has been done in [1]. Calculations at finite $T$ is quite similar [7,20]. The imaginary time free fermion propagator, written in the Landau gauge, is given by

$G\left(r_{1}, r_{2}\right)=\frac{1}{\beta} \sum_{i \omega_{n}} \sum_{l} \sum_{k} e^{-i \omega_{n}\left(\tau_{1}-\tau_{2}\right)} \frac{1}{L \tilde{l}_{0} 2^{l} \sqrt{\pi} l !} e^{i k\left(x_{1}-x_{2}\right)} \frac{1}{i \omega_{n}-\left(\epsilon_{l}-\tilde{\mu}\right)} \tilde{H}_{l}\left(\frac{y_{1}}{\tilde{l}_{0}}-\tilde{l}_{0} k\right) \tilde{H}_{l}\left(\frac{y_{2}}{\tilde{l}_{0}}-\tilde{l}_{0} k\right)$

with

$$
\tilde{H}_{l}(y)=e^{-\frac{y^{2}}{2}} H_{l}(y)
$$

where $H_{l}(y)$ is a Hermit polynomial. The one-loop kernel in real space may be written as 


$$
\tilde{\Pi}^{0}\left(r_{1}, r_{2}\right)=-\left(\begin{array}{cccc}
\left\langle\rho\left(r_{1}\right) \rho\left(r_{2}\right)\right\rangle & \left\langle\rho\left(r_{1}\right) j_{x}\left(r_{2}\right)\right\rangle & \left\langle\rho\left(r_{1}\right) j_{y}\left(r_{2}\right)\right\rangle & \frac{\omega_{c}}{2}\left\langle\rho\left(r_{1}\right) \rho\left(r_{2}\right)\right\rangle \\
\left\langle j_{x}\left(r_{1}\right) \rho\left(r_{2}\right)\right\rangle & \left\langle j_{x}\left(r_{1}\right) j_{x}\left(r_{2}\right)\right\rangle+\frac{\bar{\rho}}{m^{*}} & \left\langle j_{x}\left(r_{1}\right) j_{y}\left(r_{2}\right)\right\rangle & \frac{m^{*}}{m_{b}}\left\langle\rho\left(r_{1}\right) h\left(r_{2}\right)\right\rangle \\
\left\langle j_{y}\left(r_{1}\right) \rho\left(r_{2}\right)\right\rangle & \left\langle j_{y}\left(r_{1}\right) j_{x}\left(r_{2}\right)\right\rangle & \left\langle j_{y}\left(r_{1}\right) j_{y}\left(r_{2}\right)\right\rangle+\frac{\bar{\rho}}{m^{*}} & -\frac{m^{*}}{m_{b}}\left\langle j_{x}\left(r_{1}\right) h\left(r_{2}\right)\right\rangle \\
\frac{\omega_{c}}{2}\left\langle j_{y}\left(r_{1}\right) \rho\left(r_{2}\right)\right\rangle \\
\left.-\frac{m^{*}}{m_{b}}\left\langle h\left(r_{1}\right) \rho\left(r_{2}\right)\right)\right\rangle & -\frac{m^{*}}{m_{b}}\left\langle h\left(r_{1}\right) j_{x}\left(r_{2}\right)\right\rangle & -\frac{m^{*}}{m_{b}}\left\langle h\left(r_{1}\right) j_{y}\left(r_{2}\right)\right\rangle & -\frac{m^{*}}{m_{b}}\left\langle j_{y}\left(r_{1}\right) h\left(r_{2}\right)\right\rangle \\
\left.\left.\frac{\omega_{c}}{2} \rho\left(r_{2}\right)-\frac{m^{*}}{m_{b}} h\left(r_{2}\right)\right)\right\rangle
\end{array}\right)
$$

where \langle\rangle denotes time-ordered average. To make our notation compact, we express the matrix elements of $\tilde{\Pi}^{0}$ with index $\mu \nu$, such that for $\mu, \nu=0,1,2$, they represent the fluctuating gauge field $a_{\mu}$, while for $\mu, \nu=3$, they represent the fluctuating $\lambda$-field. Now, as an example, we work out $\tilde{\Pi}_{11}$ in detail.

$$
\begin{aligned}
\tilde{\Pi}_{11}\left(r_{1}, r_{2}\right)= & -\frac{1}{4 m_{b}^{2}}\left\{\left[D_{x_{1}} G\left(r_{1}-r_{2}\right)\right] D_{x_{2}} G\left(r_{2}-r_{1}\right)+\left[D_{x_{2}}^{\dagger} G\left(r_{1}-r_{2}\right)\right] D_{x_{1}}^{\dagger} G\left(r_{2}-r_{1}\right)\right. \\
& \left.-\left[D_{x_{1}} D_{x_{2}}^{\dagger} G\left(r_{1}-r_{2}\right)\right] G\left(r_{2}-r_{1}\right)-G\left(r_{1}-r_{2}\right) D_{x_{1}}^{\dagger} D_{x_{2}} G\left(r_{2}-r_{1}\right)\right\} \\
& +\delta^{3}\left(r_{1}-r_{2}\right) \frac{\bar{\rho}}{m^{*}}
\end{aligned}
$$

where $\vec{D}$ is the covariant derivative. In the Landau gauge it has the form

$$
\vec{D}=\vec{\nabla}+i \frac{e}{c} \vec{A}=\hat{x}\left(\partial_{x}-i \frac{B e}{c} y\right)+\hat{y} \partial_{y}
$$

Define the Fourier transform

$$
\tilde{\Pi}_{\mu \nu}^{0}\left(\vec{q}, i \omega_{n} ; \vec{q}^{\prime}, i \omega_{n}^{\prime}\right)=\int_{0}^{\beta} d \tau_{1} \int_{0}^{\beta} d \tau_{2} \int d^{2} r_{1} d^{2} r_{2} e^{-i\left(\vec{q} r_{1}-\omega_{n} \tau_{1}\right)} e^{-i\left(\vec{q}^{\prime} \vec{r}_{2}-\omega_{n}^{\prime} \tau_{2}\right)} \tilde{\Pi}_{\mu \nu}^{0}\left(r_{1}, r_{2}\right)
$$

With the above expression of the free fermion propagator, we have

$$
\begin{aligned}
& \tilde{\Pi}_{11}\left(\vec{q}, i \omega_{n} ; \vec{q}^{\prime}, i \omega_{n}^{\prime}\right)=\delta\left(q_{x}+q_{x}^{\prime}\right) \delta\left(i \omega_{n}+i \omega_{n}^{\prime}\right) \\
& \left\{\left(-\frac{1}{4 m_{b}^{2}}\right) \sum_{l, l^{\prime}} \sum_{k} \sum_{i \nu_{n}} \frac{1}{l_{0}^{2} \pi 2^{l} l ! 2^{l^{\prime}} l^{\prime} !} \frac{1}{i \nu_{n}-\left(\epsilon_{l}-\tilde{\mu}\right)} \frac{1}{\left(i \nu_{n}+i \omega_{n}\right)-\left(\epsilon_{l^{\prime}}-\tilde{\mu}\right)} I_{1 x}(\vec{q}) I_{1 x}\left(\vec{q}^{\prime}\right)+\delta\left(q_{y}+q_{y}^{\prime}\right) \frac{\bar{\rho}}{m^{*}}\right\},
\end{aligned}
$$


with

$$
\begin{aligned}
I_{1 x}(\vec{q})= & \int d y e^{-i q_{y} y}\left\{\left(i\left(k-q_{x}\right)-\frac{i}{\tilde{l}_{0}^{2}} y\right) \tilde{H}_{l}\left(\frac{y}{\tilde{l}_{0}}-\tilde{l}_{0} k\right) \tilde{H}_{l^{\prime}}\left(\frac{y}{\tilde{l}_{0}}-\tilde{l}_{0}\left(k-q_{x}\right)\right)-\right. \\
& \left.\left(-i k+\frac{i}{\tilde{l}_{0}^{2}} y\right) \tilde{H}_{l}\left(\frac{y}{\tilde{l}_{0}}-\tilde{l}_{0} k\right) \tilde{H}_{l^{\prime}}\left(\frac{y}{\tilde{l}_{0}}-\tilde{l}_{0}\left(k-q_{x}\right)\right)\right\} .
\end{aligned}
$$

In the above expressions $i \omega_{n}=i \frac{2 n \pi}{\beta}$ is the bosonic Matsubara frequency while $i \nu_{n}$ is fermionic, given by $i \frac{(2 n+1) \pi}{\beta}$. The sum over $i \nu_{n}$ may be done through the standard contour integral method [27]. The result is (for $i \omega_{n} \neq 0$ )

$$
\sum_{i \nu_{n}} \frac{1}{i \nu_{n}-\left(\epsilon_{l}-\tilde{\mu}\right)} \frac{1}{\left(i \nu_{n}+i \omega_{n}\right)-\left(\epsilon_{l^{\prime}}-\tilde{\mu}\right)}=\beta \frac{f\left(\epsilon_{l^{\prime}}-\tilde{\mu}\right)-f\left(\epsilon_{l}-\tilde{\mu}\right)}{i \omega_{n}-\left(\epsilon_{l}-\epsilon_{l^{\prime}}\right)},
$$

where $f(z)=1 /\left(e^{\beta z}+1\right)$ is the fermi distribution function. Now we compute the integral $I_{1 x}(\vec{q})$. Using the relation [28]

$$
y H_{l}(y)=\frac{1}{2} H_{l+1}(y)+l H_{l-1}(y),
$$

we have

$$
\begin{aligned}
I_{1 x}(\vec{q})= & \int d y e^{-i q_{y} y}\left(-\frac{i}{\tilde{l}_{0}}\right)\left\{\tilde{H}_{l}\left(\frac{y}{\tilde{l}_{0}}-\tilde{l}_{0} k\right)\left[\frac{1}{2} \tilde{H}_{l^{\prime}+1}\left(\frac{y}{\tilde{l}_{0}}-\tilde{l}_{0}\left(k-q_{x}\right)\right)+l^{\prime} \tilde{H}_{l^{\prime}-1}\left(\frac{y}{\tilde{l}_{0}}-\tilde{l}_{0}\left(k-q_{x}\right)\right)\right]\right. \\
& \left.+\left[\frac{1}{2} \tilde{H}_{l+1}\left(\frac{y}{\tilde{l}_{0}}-\tilde{l}_{0} k\right)+l \tilde{H}_{l-1}\left(\frac{y}{\tilde{l}_{0}}-\tilde{l}_{0} k\right)\right] \tilde{H}_{l^{\prime}}\left(\frac{y}{\tilde{l}_{0}}-\tilde{l}_{0}\left(k-q_{x}\right)\right)\right\}
\end{aligned}
$$

Shifting the variable $y$, and use [28]

$$
\int e^{-x^{2}} H_{m}(x+y) H_{l}(x+z) d x=\sqrt{\pi} 2^{l} m ! z^{l-m} L_{m}^{l-m}(-2 y z), \quad m \leq l
$$

we have

$$
\begin{aligned}
I_{1 x}(\vec{q})= & \left(-\frac{i}{\tilde{l}_{0}}\right) e^{-i q_{y} \tilde{l}_{0}^{2}\left(k-\frac{q_{x}}{2}\right)} e^{-\frac{\tilde{i}_{0}^{2} q^{2}}{4}} \pi \tilde{l}_{0} 2^{l^{\prime}} l !\left(\frac{\tilde{l}_{0}\left(q_{x}-i q_{y}\right)}{2}\right)^{l^{\prime}-l-1} \\
& \left.\left\{\frac{\tilde{l}_{0}^{2}\left(q_{x}-i q_{y}\right)^{2}}{4} L_{l}^{l^{\prime}-l+1}(x)+\frac{l^{\prime}}{2} L_{l}^{l^{\prime}-l-1}(x)+\frac{1}{2}(l+1) L_{l+1}^{l^{\prime}-l-1}(x)+\frac{\tilde{l}_{0}^{2}\left(q_{x}-i q_{y}\right)^{2}}{4}\right) L_{l-1}^{l^{\prime}-l+1}(x)\right\}, \\
& \left(l<l^{\prime}\right) ; \\
= & \left(l \leftrightarrow l^{\prime}, \quad q_{x}-i q_{y} \leftrightarrow-\left(q_{x}+i q_{y}\right)\right), \\
& \left(l>l^{\prime}\right) .
\end{aligned}
$$


Here $x \equiv \tilde{l}_{0}^{2} q^{2} / 2$. After some algebra, we obtain

$$
\begin{aligned}
\frac{\tilde{l}_{0}\left(q_{x}+i q_{y}\right)}{2} I_{1 x}(\vec{q})= & \left(-\frac{i}{\tilde{l}_{0}}\right) e^{-i q_{y} \tilde{l}_{0}^{2}\left(k-\frac{q_{x}}{2}\right)} e^{-\frac{\tilde{l}_{0}^{2} q^{2}}{4}} \pi \tilde{l}_{0} 2^{l^{\prime}} l !\left(\frac{\tilde{l}_{0}\left(q_{x}-i q_{y}\right)}{2}\right)^{l^{\prime}-l-1} \\
& \left\{q_{x}\left(l^{\prime}-l\right) L_{l}^{l^{\prime}-l}(x)+i q_{y}\left[2 x \frac{d L_{l}^{l^{\prime}-l}(x)}{d x}+\left(l^{\prime}-l-x\right) L_{l}^{l^{\prime}-l}(x)\right]\right\}, \quad\left(l<l^{\prime}\right) ; \\
-\frac{\tilde{l}_{0}\left(q_{x}-i q_{y}\right)}{2} I_{1 x}(\vec{q})= & \left(\frac{i}{\tilde{l}_{0}}\right) e^{-i q_{y} \tilde{l}_{0}^{2}\left(k-\frac{q_{x}}{2}\right)} e^{-\frac{\tilde{i}_{0}^{2} q^{2}}{4}} \pi \tilde{l}_{0} 2^{l} l^{\prime} !\left(-\frac{\tilde{l}_{0}\left(q_{x}+i q_{y}\right)}{2}\right)^{l-l^{\prime}-1} \\
& \left\{q_{x}\left(l-l^{\prime}\right) L_{l^{\prime}}^{l-l^{\prime}}(x)-i q_{y}\left[2 x \frac{d L_{l^{\prime}}^{l-l^{\prime}}(x)}{d x}+\left(l-l^{\prime}-x\right) L_{l^{\prime}}^{l-l^{\prime}}(x)\right]\right\}, \quad\left(l>l^{\prime}\right) ;
\end{aligned}
$$

The summation over $k$ may be straightforwardly carried out

$$
\sum_{k} e^{i \tilde{l}_{0}^{2} k\left(q_{y}+q_{y^{\prime}}\right)}=\frac{L^{2}}{2 \pi \tilde{l}_{0}^{2}} \delta\left(q_{y}+q_{y}^{\prime}\right)
$$

where $\frac{L^{2}}{2 \pi I_{0}^{2}}$ is the degeneracy of a (pseudo) Landau level. These lead to

$$
\begin{gathered}
\tilde{\Pi}_{11}^{0}\left(\vec{q}, i \omega_{n} ; \vec{q}^{\prime}, i \omega_{n}^{\prime}\right)=\delta^{2}\left(\vec{q}+\vec{q}^{\prime}\right) \delta\left(i \omega_{n}+i \omega_{n}^{\prime}\right)( \\
\frac{L^{2} \beta}{2 \pi \tilde{\omega}_{c}} e^{-x} \sum_{m<l} \frac{l-m}{\left(\frac{i \omega_{n}}{\tilde{\omega}_{c}}\right)^{2}-(l-m)^{2}} \frac{m !}{l !} x^{l-m-1}\left\{f\left(\epsilon_{m}-\tilde{\mu}\right)-f\left(\epsilon_{l}-\tilde{\mu}\right)\right\} \\
\left\{\left(i \omega_{n}\right)^{2}\left(L_{m}^{l-m}(x)\right)^{2}+x\left[2 \frac{d L_{m}^{l-m}(x)}{d x}-L_{m}^{l-m}(x)\right]\left[(2(l-m)-x) L_{m}^{l-m}(x)+2 x \frac{d L_{m}^{l-m}(x)}{d x}\right] \tilde{\omega}_{c}^{2} \frac{q_{y}^{2}}{q^{2}}\right\} \\
\left.-\frac{L^{2} \beta}{2 \pi \tilde{\omega}_{c}} e^{-x} \sum_{m<l} \frac{m !}{l !} x^{l-m-1}(l-m)\left\{f\left(\epsilon_{m}-\tilde{\mu}\right)-f\left(\epsilon_{l}-\tilde{\mu}\right)\right\} \tilde{\omega}_{c}^{2}\left(L_{m}^{l-m}(x)\right)^{2}+\frac{\bar{\rho}}{m^{*}}\right) .
\end{gathered}
$$

Define

$$
\tilde{\Pi}^{0}\left(\vec{q}, i \omega_{n} ; \vec{q}^{\prime}, i \omega_{n}^{\prime}\right) \equiv \delta\left(q_{x}+q_{x}^{\prime}\right) \delta\left(i \omega_{n}+i \omega_{n}^{\prime}\right) \Pi^{0}\left(\vec{q}, i \omega_{n}\right)
$$

we have

$$
\begin{aligned}
\Pi_{11}^{0}\left(\vec{q}, i \omega_{n}\right)= & \frac{\tilde{n}}{2 \pi \tilde{\phi}}\left\{\frac{i \omega_{n}^{2}}{\tilde{\omega}_{c}} \Sigma_{0}+\tilde{\omega}_{c} \frac{q_{y}^{2}}{q^{2}} \Sigma_{3}\right\}- \\
& \frac{\tilde{n}}{2 \pi \tilde{\phi}} \tilde{\omega}_{c} \frac{e^{-x}}{n} \sum_{m<l} \frac{m !}{l !} x^{l-m-1}(l-m)\left[f\left(\epsilon_{m}-\tilde{\mu}\right)-f\left(\epsilon_{l}-\tilde{\mu}\right)\right] .
\end{aligned}
$$

The last term vanishes as $x \rightarrow 0$. According to [1], we ignore it hereafter. (A similar treatment is also applied to $\Pi_{22}^{0}$.) This ensures the gauge invariance of $\Pi^{0}$. The new matrix elements which appear in the present theory are those involve the (mean-field) kinetic energy density $h(r)$. Now we evaluate one of them as an example. Consider 


$$
\begin{aligned}
\tilde{\Pi}_{03}\left(r_{1}, r_{2}\right)= & \frac{\omega_{c}^{b}}{2} G\left(r_{1}-r_{2}\right) G\left(r_{2}-r_{1}\right)+ \\
& \frac{1}{4 m_{b}}\left\{G\left(r_{1}-r_{2}\right) \vec{D}_{\vec{r}_{2}}^{2} G\left(r_{2}-r_{1}\right)+\left[\vec{D}_{\vec{r}_{2}}^{\dagger 2} G\left(r_{1}-r_{2}\right)\right] G\left(r_{2}-r_{1}\right)\right\}
\end{aligned}
$$

In the Fourier space we have

$$
\begin{aligned}
\tilde{\Pi}_{03}\left(\vec{q}, i \omega_{n} ; \vec{q}^{\prime}, i \omega_{n}^{\prime}\right)= & \delta\left(q_{x}+q_{x}^{\prime}\right) \delta\left(i \omega_{n}+i \omega_{n}^{\prime}\right) \sum_{l, l^{\prime}} \sum_{k} \sum_{i \nu_{n}} \frac{1}{\tilde{l}_{0}^{2} \pi 2^{l} l ! 2^{l^{\prime}} l^{\prime} !} \\
& \frac{1}{i \nu_{n}-\left(\epsilon_{l}-\tilde{\mu}\right)} \frac{1}{\left(i \nu_{n}+i \omega_{n}\right)-\left(\epsilon_{l^{\prime}}-\tilde{\mu}\right)}\left(\frac{\omega_{c}^{b}}{2}-\frac{l+l^{\prime}+1}{2 m_{b} \tilde{l}_{0}^{2}}\right) I_{0}(\vec{q}) I_{0}\left(\vec{q}^{\prime}\right)
\end{aligned}
$$

with

$$
I_{0}(\vec{q})=\int d y e^{-i q_{y} y} \tilde{H}_{l}\left(\frac{y}{\tilde{l}_{0}}-\tilde{l}_{0} k\right) \tilde{H}_{l^{\prime}}\left(\frac{y}{\tilde{l}_{0}}-\tilde{l}_{0}\left(k-q_{x}\right)\right)
$$

$I_{0}$ may be evaluated straightforwardly using (72). The final result is given in (21)

Now we consider the case $i \omega_{n}=0$. For $l^{\prime} \neq l$, the Matsubara frequency sum in (67) is still given by (69) with substitution $i \omega_{n}=0$. However, the term with $l^{\prime}=l$ also contribute in the present situation:

$$
\sum_{i \nu_{n}} \frac{1}{\left[i \nu_{n}-\left(\epsilon_{l}-\tilde{\mu}\right)\right]^{2}}=\left.\beta \frac{\partial f(z)}{\partial z}\right|_{z=\epsilon_{l}-\tilde{\mu}}=-\beta^{2} e^{\left(\epsilon_{l}-\tilde{\mu}\right)} f^{2}\left(\epsilon_{l}-\tilde{\mu}\right)
$$

To evaluate, e.g., $\Pi_{11}^{0}(q, 0)$, one needs calculate the integral (68) for the case $l=l^{\prime}$. Using a similar procedure, one obtains

$$
\left.I_{1 x}(\vec{q})\right|_{l=l^{\prime}}=q_{y} e^{-i q_{y} \tilde{l}_{0}^{2}\left(k-\frac{q_{x}}{2}\right)} e^{-\frac{\tilde{i}_{0}^{2} q^{2}}{4}} \pi \tilde{l}_{0} 2^{l} l !\left[L_{l}^{0}(x)-2 L_{l}^{1}(x)\right]
$$

This result, together with that obtained for $l \neq l^{\prime}$, eqn. (73), gives $\Pi_{11}^{0}(q, 0)$ expressed in (28). Other matrix elements in (21) and (28) may be obtained in similar manner. 


\section{REFERENCES}

[1] A. Lopez and E. Fradkin, Phys. Rev. B 445246 (1991); ibid, 477080 (1993).

[2] B. I. Halperin, P. A. Lee, and N. Read, Phys.Rev.B 47, 7312 (1993).

[3] J.K.Jain, Phys.Rev.Lett., 63, 199 (1989); Phys.Rev.B 41, 7653 (1990); Adv. Phys. 41, 105 (1992).

[4] For a review, see, e.g., The Quantum Hall Effect, eds. R.E.Prange and S.M.Girvin, Springer-Verlag, New York, 1990.

[5] S.H.Simon and B.I.Halperin, Phys. Rev. B 48, 17368 (1993); ibid, 501807 (1994).

[6] S. He, S.H.Simon and B.I.Halperin, Phys. Rev. B 501823 (1994).

[7] L. Zhang, Phys. Rev. B 51, 4645 (1995).

[8] R.L. Willett et al, Phys. Rev. Lett. 65, 112 (1990); Phys. Rev. B47, 7344 (1993).

[9] W. Kang et al., Phys. Rev. Lett. 71, 3850 (1993); V.J. Goldman et al., ibid, 72, 2065 (1994); R.R. Du et al., ibid, 70, 2944 (1993); ibid, 733274 (1994); Solid State Commun. 90, 71 (1994); D.R. Leadley et al., Phys. Rev. Lett. 72, 1906 (1994); R.L. Willett et al., ibid, 71, 3846 (1993); H.C. Manoharan et al., ibid, 73, 3270 (1994).

[10] R.B. Laughlin Phys. Rev. Lett. 501395 (1983).

[11] R.K. Kamilla and J.K. Jain, unpublished.

[12] B. Sakita, D.N. Sheng and Z.B. Su, Phys. Rev. B 44, 11510 (1991); Z.S. Ma and Z.B. Su, ibid, 482347 (1993); R. Ray and B. Sakita, Ann. Phys. (N.Y.) 230, 131 (1994); R. Ray and G. Gat, unpublished.

[13] J. Martinez and M. Stone, Int. J. Mod. Phys. B 7, 4389 (1993); R. Rajaraman, ibid, 8 777 (1994); R. Rajaraman and S.L. Sondhi, unpublished.

[14] R.R.Du , H.L.Stormer, D.C.Tsui, L.N.Pfeiffer, and K.W.West, Phys.Rev.Lett. 70, 2944 
(1993).

[15] L.L. Sohn et al, Solid State Comm., 93897 (1995).

[16] S.M. Girvin, A.H. MacDonald and P.M. Platzman, Phys.Rev.Lett., 54, 581 (1985); Phys.Rev.B, 33, 2481 (1986).

[17] S.C. Zhang, H. Hansson and S. Kivelson, Phys. Rev. Lett. 62, 82 (1989); D.H. Lee and S.C. Zhang, ibid 66, 1220 (1991).

[18] Y.B. Kim, A. Furusaki, X.G. Wen and P.A. Lee, Phys. Rev. B 50, 17917 (1994); Y.B. Kim, P.A. Lee, X.G. Wen and P.C.E. Stamp, unpublished.

[19] S.E. Barnes, J. Phys. F, 6, 1375 (1976); P. Coleman, Phys. Rev. B, 29, 3035 (1984); N. Read and D.M. Newns, J. Phys. C, 16, 3273 (1983).

[20] S. Randjbar-Daemi, A. Salam and J. Strathdee, Nucl. Phys. B340, 403 (1990).

[21] B.I. Halperin, T.C. Lubensky and S.K. Ma, Phys. Rev. Lett. 32, 292 (1974)

[22] J.R. Schrieffer, Theory of Superconductivity, Benjamin-Cummings, 3rd printing (1983).

[23] S. Coleman and E. Weinberg, Phys. Rev. D 7, 1988 (1973).

[24] Z.Y. Weng, Phys. Rev. Lett. 66, 2156 (1991).

[25] W. Kohn, Phys. Rev. 123, 1242 (1961).

[26] Although $\Sigma_{2}$ does not appear in $\Pi^{0}$, we still show it here and in the subsequent calculations. $\Sigma_{2}$ emerges when one adopts the special guage choice $\vec{\nabla} \cdot \vec{a}=0$, with $\vec{a}_{T} \| \hat{y}$ and $\vec{q} \| \hat{x}$, as it was done in [5] for the unconstrained case. While the two calculations coincide there in the $q \rightarrow 0$ limit, we find that they differ at finite $q$, contrary to what was found in [5]. The physical origin of this discrepancy is not clear, although one may argue that it is due to the "weak form" [] of the gauge invariance property of $\Pi^{0}$. They would give the same collective modes (i.e., the same poles in $K_{00}$ ) if one 
substitutes $\left(\frac{i \omega_{n}}{\tilde{\omega}_{c}}\right)^{2} \Sigma_{0}+\Sigma_{3}$ with $\Sigma_{2}+1$. One may also make such a substitution in (35) for the constraint theory. Notice that such a problem does not occur in the $\nu=1 / 2$ case considered in [2] where the mean-field ground state is a filled Fermi-sea.

[27] G.D. Mahan, Many Particle Physics Plenum, 2nd Edition, (1990).

[28] I.S. Gradshteyn and I.M. Ryzhik, Tables of Integrals, Series, and Products, Academic Press, (1980). 


\section{FIGURES}

Some mean field ground state configurations at $\nu=1 / 3$ in the theory with the LLL

constraint. $E$ is in the unit of $\hbar \bar{\omega}_{c}$. In the configuration (a), all the fermions are filled into the first LL to satisfy (9). However, there are other ways of placing fermions which also produce homogeneous states satisfying (9), such as those shown in (b),(c) and (d), in which some fermions are placed in the 0-th LL and higher LLs. The degeneracy of the mean field ground state becomes infinite in the thermal dynamical limit. 


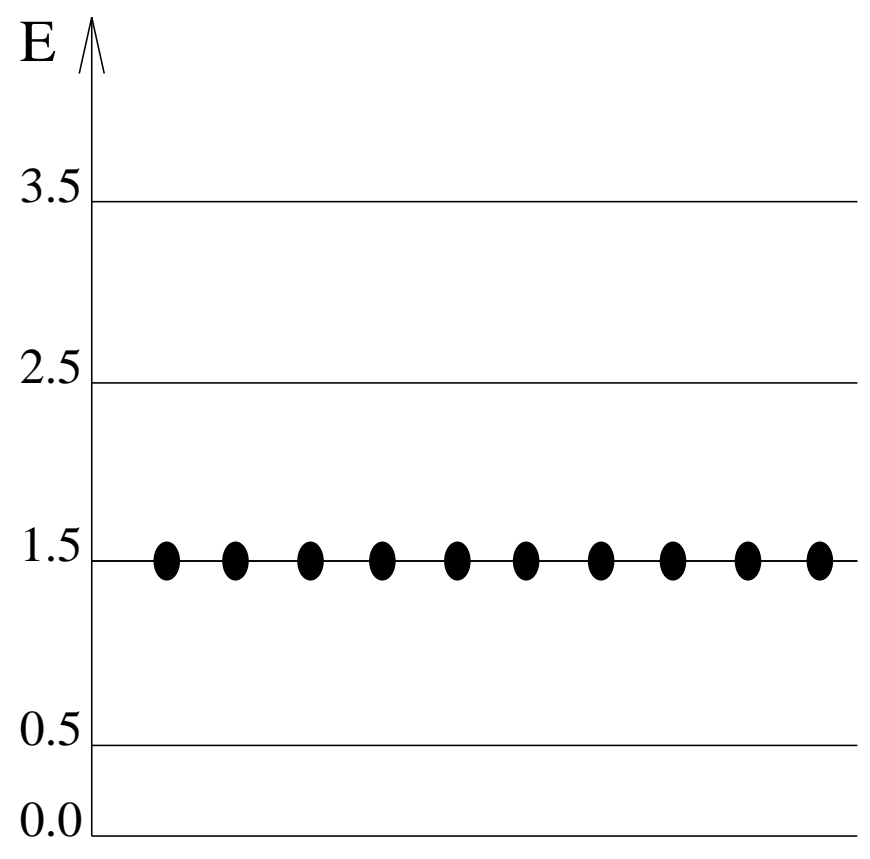

(a)

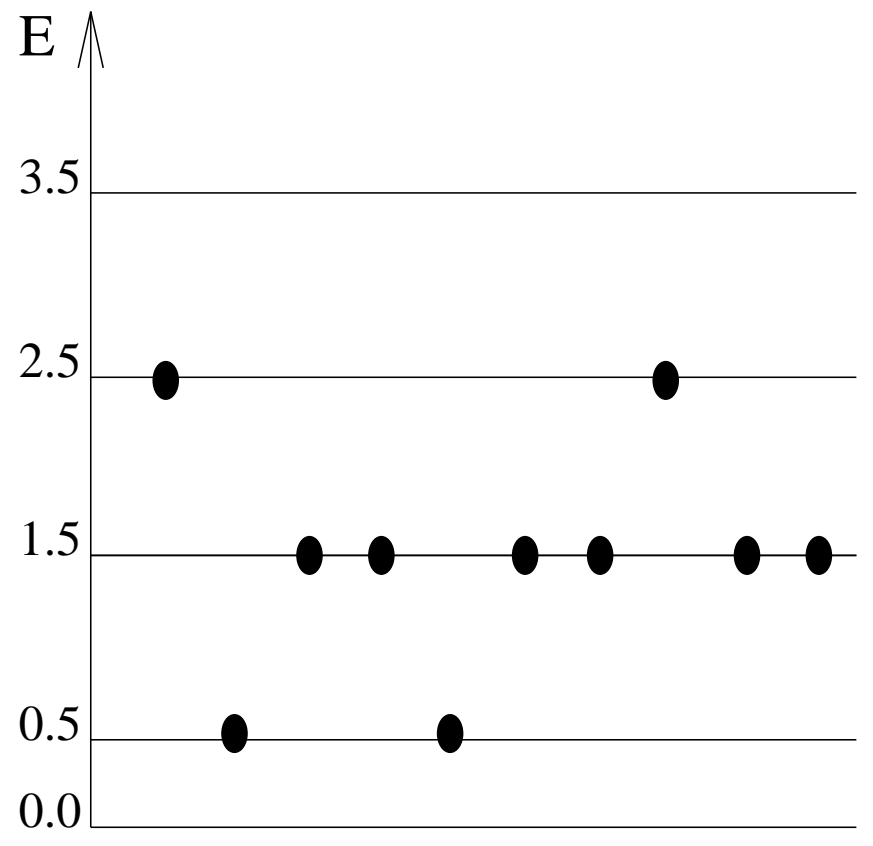

(c)

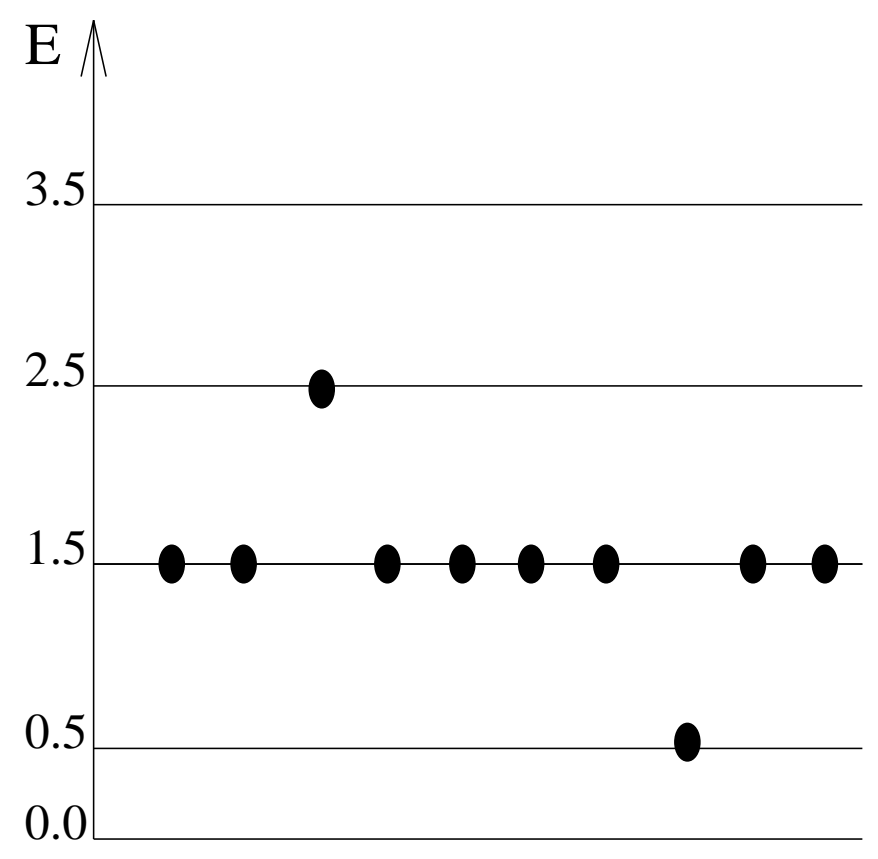

(b)

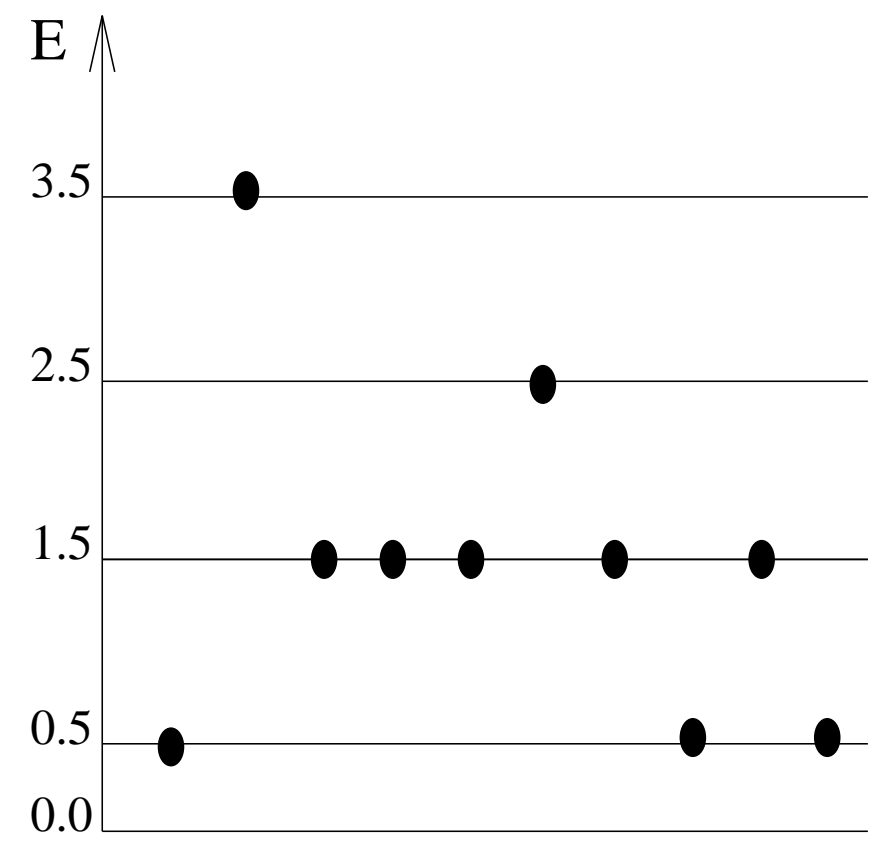

(d)

Figure 1 